\title{
Coordinated Toolpath Planning for Multi-Extruder Additive Manufacturing
}

This paper was downloaded from TechRxiv (https://www.techrxiv.org).

\section{LICENSE}

CC BY 4.0

SUBMISSION DATE / POSTED DATE

07-10-2021 / 25-10-2021

\section{CITATION}

Khatkar, Jayant; Yoo, Chanyeol; Fitch, Robert; Clemon, Lee; Mettu, Ramgopal (2021): Coordinated Toolpath Planning for Multi-Extruder Additive Manufacturing. TechRxiv. Preprint.

https://doi.org/10.36227/techrxiv.16764451.v1

$\mathrm{DOI}$

10.36227/techrxiv.16764451.v1 


\title{
Coordinated Toolpath Planning for Multi-Extruder Additive Manufacturing
}

\author{
Jayant Khatkar ${ }^{1}$, Chanyeol Yoo ${ }^{1}$, Robert Fitch ${ }^{1}$, Lee Clemon ${ }^{1}$, Ramgopal Mettu ${ }^{2}$
}

\begin{abstract}
We present a new algorithm for coordinating the motion of multiple extruders to increase throughput in fused filament fabrication (FFF)/fused deposition modelling (FDM) additive manufacturing. Platforms based on FFF are commonly available and advantageous to several industries, but are limited by slow fabrication time and could be could be significantly improved through efficient use of multiple extruders. We propose the coordinated toolpath planning problem for systems of extruders mounted as end-effectors on robot arms with the objective of maximising utilization and avoiding collisions. Building on the idea of dependency graphs introduced in our earlier work, we develop a planning and control framework that precomputes a set of multi-layer toolpath segments from the input model and efficiently assigns them to individual extruders such that executed toolpaths are collision-free. Our method overcomes key limitations of existing methods, including utilization loss from workspace partitioning, precomputed toolpaths subject to collisions with the partially fabricated object, and wasted motion resulting from strict layer-by-layer fabrication. We report simulation results that show a major increase in utilization compared to single- and multi-extruder methods, and favorable fabrication results using commodity hardware that demonstrate the feasibility of our method in practice.
\end{abstract}

Note to Practioners-As additive manufacturing continues to proliferate, production planning and optimization needs will become more critical. This work presents a framework that seeks to maximize concurrency of multiple independent extruders operating in a shared workspace. Given an input model, our system maximizes the speed of fabrication and automatically generate toolpaths at runtime. This approach adapts to the geometry of a given part instead of slicing the input model and fabricating it in a strict layer-by-layer sequence. Importantly, our framework is also general enough to allow for multiple specialized extruders that, for example, utilize different materials or operate with different physical capabilities.

Index Terms-Additive Manufacturing (AM), Multi-arm coordination, process planning.

\section{INTRODUCTION}

Additive manufacturing has emerged as a next-generation technology for advanced fabrication. While there are a variety of additive manufacturing methods and materials available, fused deposition modeling (FDM), also known as fused filament fabrication (FFF) or material extrusion [1], is highly versatile and is the most routine method in use, in part due to the availability and low cost of materials and necessary

This research is supported by an Australian Government Research Training Program (RTP) Scholarship and the University of Technology Sydney.

${ }^{1}$ School of Mechanical and Mechatronic Engineering, University of Technology Sydney, Australia jayant.khatkarestudent.uts.edu.au, \{chanyeol.yoo, robert.fitch, lee.clemon\}auts.edu.au.

${ }^{2}$ Department of Computer Science, Tulane University, USA rmettudtulane. edu. mechanical components, as well as the expiration of key patents. For these reasons, perhaps the most commonly available platforms for additive manufacturing (e.g., consumergrade systems) are based on FFF. FFF-based hardware is also ubiquitous in commercial settings, and is used in rapid prototyping due to its high quality and versatility of material.

Despite its advantages, FFF continues to be limited to custom or low-volume production because of slow fabrication time. The throughput of any FFF platform is fundamentally limited by its rate of extrusion, even though implementation varies according to cost and material constraints. Enabling FFF for large-scale additive manufacturing of high-quality parts with high production efficiency is important to manufacturing industries [2]. A basic efficiency goal is to maximize manufacturing throughput, that is, the amount of fabrication achievable per unit time. The necessary improvements in throughput cannot be achieved by incremental hardware advances alone. We believe that key to significantly improving fabrication time is to parallelize the process by using multiple extruders.

Multiple extruders can add material simultaneously to produce large objects quickly. This capability also can mitigate a major source of inefficiency in single-extruder systems, in which the extruder must travel between parts of the model without depositing material. Multi-extruder systems can eliminate this inefficiency by adding material to multiple areas at once without the need to travel between them. Like multi-processor architectures, multiple-extruder platforms can approach theoretically optimal throughput through suitable planning.

In order to parallelize FFF for faster throughput, it is necessary to address fundamental algorithmic challenges that have not yet been adequately explored. A system with multiple extruders working in parallel must coordinate their motion to avoid collisions and minimize fabrication time. A distinctive property of this problem is that any particular extruder moving along a given path is subject to collision with other extruders as well as the object itself as its geometry changes during fabrication. This risk of collision leads to a phenomenon we refer to as blocking, where a given extruder is unable to execute an extrusion without colliding with another extruder currently blocking its path. If the given extruder simply waits for the other to complete its extrusion action, the resulting waiting time compounds over the duration of the plan and negatively effects total fabrication time, which is the main objective to be minimized.

Existing methods for collision avoidance use zone blocking approaches where either the workspace or model is partitioned a priori [3, 4]. Partitioned regions are mapped to extruders and 
chosen such that extruder motions are unlikely to mutually interfere. Potential collisions near partition boundaries are avoided through time delays. The intent is to maximize the utilization of each extruder, and thus minimize fabrication time, while minimizing the need for motion coordination online. However, zone blocking approaches can suffer from large penalties in utilization at the boundaries of regions allocated to each extruder, where it may only be possible to avoid collisions by blocking all but one. Moreover, existing methods operate by "slicing" the input model into a set of horizontal layers and fabricating each layer in sequence, and do not take advantage of the utilization increase made possible by fabricating multiple layers in different parts of the input model at once. Another fundamental issue is that zone blocking methods introduce arbitrary internal adhesion layers at the boundary between partitions [5], which creates unnecessary structural weaknesses; it is well known that interlayer adhesion is the primary failure mode in FFF-generated structures [6]. To maximize extruder utilization without these drawbacks, we develop a zone-free method for toolpath planning that coordinates extruder motion online.

In this paper, we consider system designs where extruders are mounted as end-effectors on 6-DOF robot arms as shown in Fig. 1. Robot arm-based systems capture a general case of extruder actuation mechanisms and facilitates ease of experimentation using off-the-shelf components. They also provide flexibility in choosing the required number of degrees of freedom, in sharp contrast to gantry-based platforms.

We formulate the coordinated toolpath planning problem and present an online algorithmic framework that decomposes the input model into a set of sub-components and schedules them for fabrication, with the objective of maximizing utilization (or equivalently, minimizing waiting time) while also satisfying collision constraints and maintaining the property that all incomplete parts of the model remain reachable. During fabrication, each extruder iteratively selects a subcomponent to fabricate based on the state of the other extruders in the system. Collision checking is performed in a novel, lazy fashion and enables toolpaths to be generated in real time.

In order to work across multiple layers, our approach utilizes the notion of a dependency graph in a 3D model, first devised in [7]. The dependency graph defines the required precedence of fabrication between sub-components of the model, ensuring that the model is correctly fabricated. We make use of it to construct toolpaths that cross multiple layers, thus allowing greater freedom in scheduling sub-components for each extruder for maximum utilization. Our method sidesteps several limitations of existing methods, including a priori allocation of work, pre-computed toolpaths, and layer-by-layer fabrication. Although the focus of this paper is on the two-extruder case, the framework is defined more generally and we expect its performance to scale well with more extruders.

We validate our approach in simulation and in hardware for the two-extruder setting. In simulation, on a benchmark of 20 models we achieve a median improvement in utilization of $76 \%$ over a single extruder and around $20 \%$ over zone blocking. Using a custom two-extruder system based on UR5e [8] commercial robot arms and commonly available

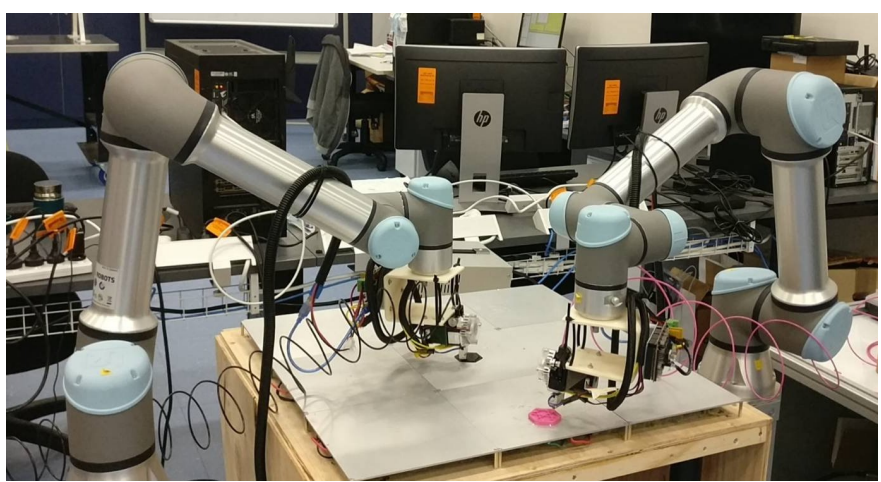

Fig. 1. Hardware implementation of our system with extruders mounted on two UR5e [8] robot arms.

FFF components (Fig. 1), we demonstrate component quality comparable to commercial systems with negligible deviation from planned fabrication time.

These experiments show substantial improvement in extruder utilization over existing methods as well as the practical potential of our approach to coordinated multi-extruder toolpath planning. A high degree of utilization across multiple extruders is a fundamental step in enabling high throughput additive manufacturing. Thus the significance of our framework and experimental results is to provide the first step towards a new platform for additive manufacturing that facilitates production-level adoption of FFF.

\section{RELATED WORK}

While most FFF systems use a gantry system to move the extruder, robotic arms have been used to enable non-planar printing [9] using the arm's additional degrees of freedom to rotate the extruder. Robot arms have also been used for large scale printing [10, 11] by mounting the robotic arm on a mobile platform. More generally, the use of robots in additive manufacturing include applications in art and construction, the reduction or elimination of support material, and the improvement in part surface quality [12]. Coordinated planning for multiple extruders actuated by serial link manipulators that operate in a shared workspace has not been explored in depth in prior work.

Existing methods for manipulator-based systems generally ignore robot arm kinematics during collision checking, and therefore cannot guarantee that the planned trajectories of individual joints are collision-free [13, 14]. This limitation impedes experimentation with real robot hardware and specifically precludes the type of coordinated planning we propose, where robot arms must operate in a shared workspace. Approaches that have been implemented in hardware apply ideas such as zone blocking to create distinct, non-overlapping workspaces [11]. Similar approaches include blocking via time delays [15], scheduling [16, 17], and online collision avoidance [18]. Our method avoids these constraints by planning coordinated paths in a shared workspace, thus enabling the major increase in extruder utilization we demonstrate experimentally.

In prior work [7, 19, 21], we presented a sequence of algorithms that seek to minimize the wasted motion or fabrication 
time of the extrusion process for a given input model. Our key insight was to observe that fabrication in a layer-by-layer fashion induces motion that can be eliminated by fabricating multiple layers in different parts of the model asynchronously. In order to guarantee that such an approach always results in a feasible toolpath, this work introduced the notion of a dependency graph computed from the input model and extruder geometry. We showed that a greedy algorithm can reduce wasted motion by around $30 \%$, whereas a more sophisticated local search algorithm can eliminate an additional 30\%. This paper applies the dependency graph concept to the case of multi-extruder coordination.

The computational complexity of planning an optimal toolpath in a single layer with a single extruder is $\mathcal{N} \mathcal{P}$-hard, since it corresponds to an instance of the Traveling Salesperson problem. Recent work in planning for coordinated gantry-based systems uses approximate methods such as handtuned heuristics [22], tabu search [5], and evolutionary algorithms [4]. Our framework uses an efficient search procedure for planning along with efficient, online collision checking.

\section{Problem Statement}

The coordinated toolpath planning problem is defined for an FFF system composed of a set of $K$ manipulator arms that collectively must fabricate a part in a shared workspace. The end effector of each arm is an extrusion mechanism. Arms are required to avoid collision with each other as well as with the partially fabricated object, and must also avoid self-collision. In this section, we define our assumptions and present the problem formally.

A given input model is sliced into 2D layers, and each layer is decomposed into individual, printable contours $\mathcal{C}$ (i.e., collections of contiguous line segments) using a standard approach (e.g., [23]). Throughout this paper we view a contour as the smallest extrudable component of a model: the extruder is placed at the first end point of a contour, emits material as it moves along the contour, and stops at the second end point.

A toolpath $\pi_{k}: C \rightarrow \mathbb{N}$ is an ordered set of contours for arm $k$. Unlike in the case of a single extruder, which must visit all contours exactly once, we must assign contours to extruders such that every contour is visited exactly once. That is, $\pi_{i} \bigcap \pi_{j}=\emptyset$ for all pairs of $i$ and $j$ toolpaths. Additionally, each arm has the option to travel to a base configuration that ensures that no other arm can collide with it. For simplicity we use the same base configuration for all arms, and denote it as $\mathcal{H}$.

A trajectory $\mathbf{x}_{c}^{k}$ is defined as a timed sequence of arm joint configurations for arm $k$ given contour $c \in \mathcal{C}$, such that the extruder follows the contour segment. The overall trajectory required for arm $k$ to follow all contours in toolpath $\pi$ is denoted as $\mathbf{x}_{\pi}^{k}$. We let $\hat{\mathbf{X}}_{c}^{k}$ denote the set of candidate trajectories for arm $k$ to print contour $c \in \mathcal{C}$. The candidate trajectories for a set of contours is denoted $\hat{\mathbf{X}}_{\mathcal{C}}^{k}$. We use $\mathbf{x}_{\pi, c}^{k}$ (or $\mathbf{x}_{c, \mathcal{H}}^{k}$ ) to denote a trajectory that moves extruder $k$ from the last contour of $\pi$ to the beginning of contour $c$; we will refer to this motion as extrusionless travel. We will let $D\left(\mathbf{x}_{\pi, c}^{k}\right)$ denote the length of the trajectory $\mathbf{x}_{\pi, c}^{k}$ in the configuration space of extruder $k$.
Given a set of $K$ extruders, we wish to find a set of $K$ trajectories, one per extruder, to collectively fabricate all contours $\mathcal{C}$ in a given 3D input model. The set of trajectories for all $K$ extruders is referred to as collective trajectory $\mathbf{X}=\left\{\mathbf{x}_{\pi_{k}}^{k}\right\}_{k}^{K}$.

Our objective is to maximize the utilization $\sigma: \mathbf{X} \rightarrow \mathbb{R}^{+}$ of a collective trajectory, which is defined as the ratio of time spent extruding by all arms and the total time, start to finish, of fabrication. The utilization a single extruder system has a theoretical maximum value of 1 , whereas with two arms the maximum value is 2 . Defining the maximization objective in this way accounts for variation in fabrication time across input models and thus facilitates performance evaluation. Fabrication times vary based on the model's external and internal geometry in addition to its orientation, and thus is not proportional to utilization. Fabrication time is also not directly proportional to the number of extruders; time improvements can be superlinear in the number of extruders for input models that induce a large amount of extrusionless travel by a single extruder.

The coordinated toolpath planning problem is to find collective trajectory $\mathbf{X}$ such that the utilization of the extruders is maximized. This maximization objective is analogous to parallel scheduling and differs from our prior work in singleextruder toolpath planning [7, 19, 21], in which we minimized wasted motion (i.e., extrusionless travel). The collective trajectory can be built incrementally as it is executed, as we do in the solution presented in this paper.

\section{Multi-Extruder CoOrdination}

In this section, we present an algorithmic framework that solves the coordinated toolpath planning problem. Our framework allows all extruders to print any contours that are in reach without any explicitly defined zones. Instead of computing a sequence of contours for each extruder offline, we perform collision checking and make assignment decisions online.

The framework broadly consists of three components: 1) preprocessing, 2) planning, 3) actuation (Fig. 2). First, an input model is preprocessed to compute the set of contours and its associated dependency graph following the procedure given in [7]. The dependency graph defines constraints on the order in which individual contours can be completed and is computed by evaluating sets of candidate trajectories. The dependency graph and the candidate trajectories are used in the planning component of the framework to iteratively generate trajectories which are executed immediately by the actuation component. Collision-checking is performed using an efficient online algorithm.

\section{A. Preprocessing The Input Model}

The object to be fabricated is initially represented as a mesh defining the shape and size of the model. This mesh is sliced to produce a sequence of planar horizontal layers that, when stacked vertically, approximate the object's 3D geometry. Each layer is represented as a set of contours. As defined above, a contour is a collection of contiguous line segments which represent a single extrusion. This representation is typical for FFF systems and can be generated by a number of commonly 


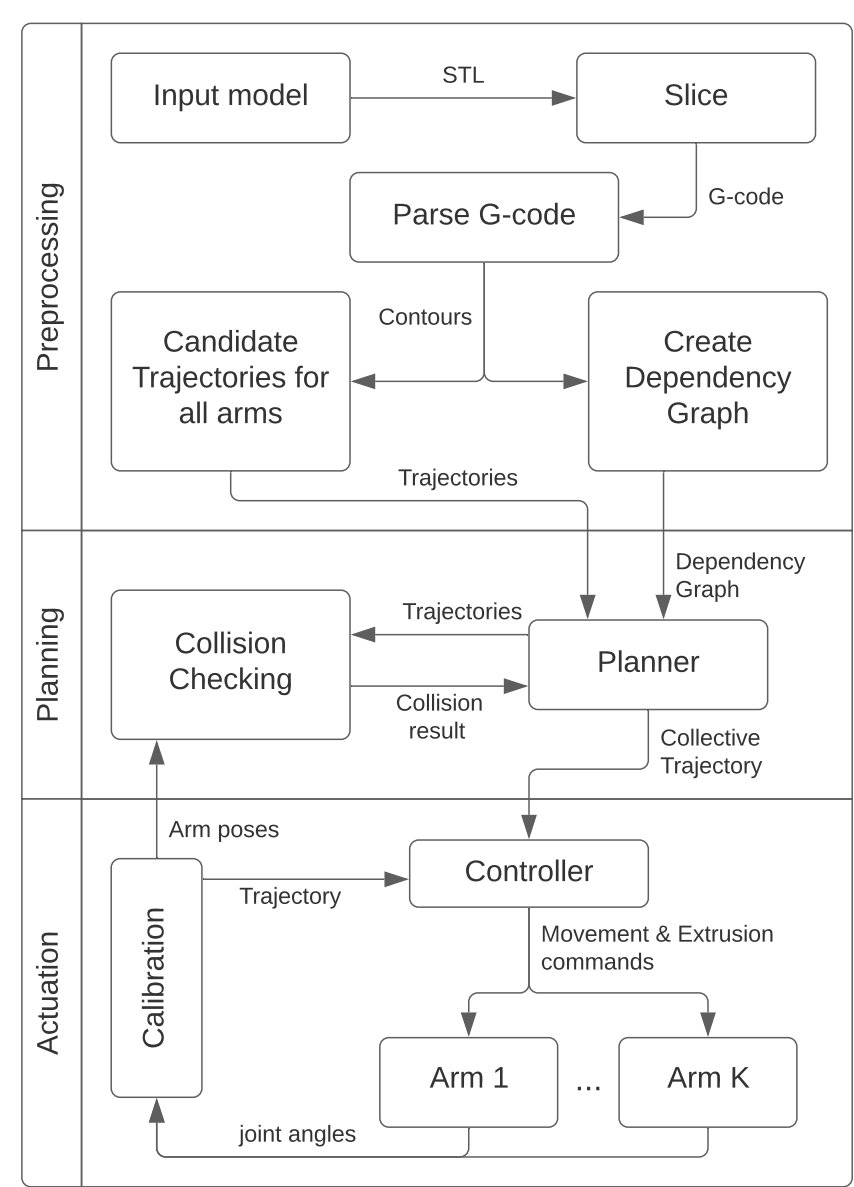

Fig. 2. High level system overview. Preprocessing creates candidate trajectories given a model, and a dependency graph over the contours to print. Planning uses these to create a collision free collective trajectory which is executed by Actuation to fabricate the model.

available software packages (e.g., in our implementation we use Sli3r [23]).

Given the total set of contours (aggregated from all layers), the first preprocessing step is to find a set of candidate trajectories for each contour and each robot arm. A candidate trajectory for a given arm is one where the extruder follows the associated contour. In general, a single end-effector position does not necessarily determine a unique joint configuration for a multi-link manipulator; this well-known inverse kinematics problem may have multiple solutions and, thus, there may exist distinct trajectories for each contour.

For contour $c \in \mathcal{C}$ and arm $k$, we assign a set of waypoints evenly along the contour and find inverse kinematic (IK) solutions for each waypoint. We limit the number of possible IK solutions by assuming that the end-effector is always orthogonal to the print bed. There are a maximum of eight unique IK solutions per waypoint for 6-DoF arms (as we consider in this paper) under this assumption. We then find sets of candidate trajectories by considering each waypoint in sequence and choosing the closest IK solution to the previous waypoint. We repeat this process for each of the initial waypoint's IK solutions.

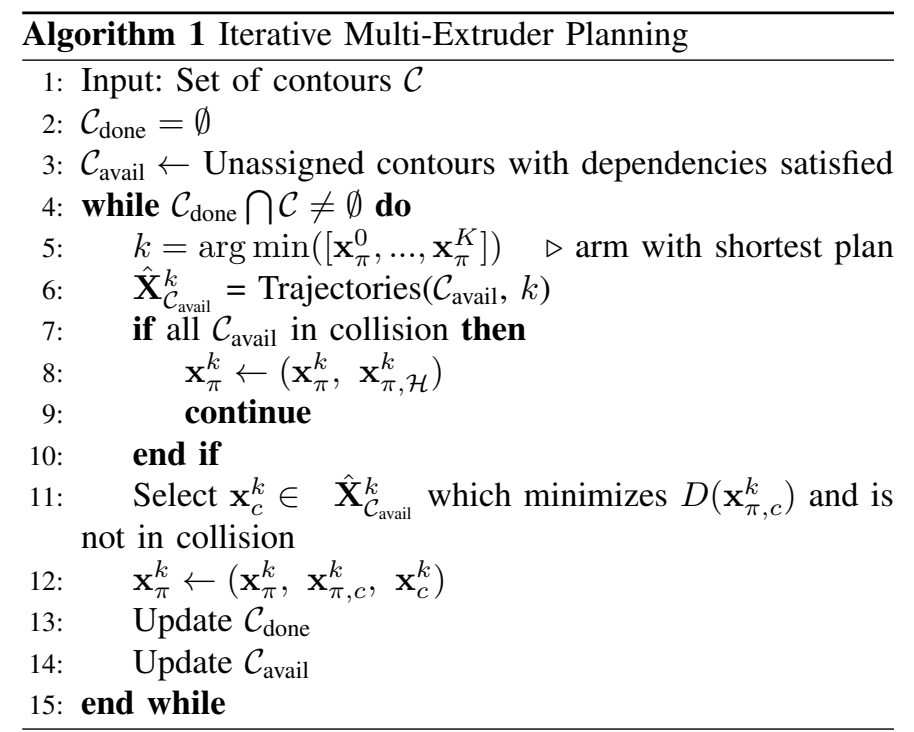

\section{B. Iterative Multi-Extruder Planning}

The planning component assigns contours to arms for immediate fabrication. Our planning algorithm attempts to assign a new contour to an arm as soon as it has finished fabricating its current contour. If no assignment is possible because all potential trajectories are in collision, the arm is commanded to return to a designated home configuration to wait for its next assignment. To assign a contour, we search for a collision-free trajectory within the set of all contours currently available for fabrication. A contour is available if all its dependencies are satisfied, which means that all of its predecessor contours have already been fabricated. These dependencies are defined in the dependency graph computed during preprocessing. Because at least one contour is available for fabrication at all times, the complete object is fabricated eventually.

The planning algorithm is presented in pseudocode in Alg. 11. The algorithm iteratively selects an extruder $k$ in priority order and attempts to assign a trajectory corresponding to one of the contours in the available set $\mathcal{C}_{\text {avail }}$. Extruders are prioritized according to the shortest time to complete the current trajectory. Idle extruders thus have highest priority. Trajectory assignment proceeds by searching the sets of trajectories associated with the available contours to find a trajectory that is collision-free. The algorithm sorts available trajectories according to shortest travel distance in the arm's configuration space, calculated by computing the distance between the last waypoint in the extruder's current trajectory and the first waypoint in the considered trajectory. The algorithm then iterates through the ranked trajectories performing collision checks. The first collision-free trajectory found is selected and its contour is assigned to the extruder for fabrication. The planning algorithm terminates when all contours have been assigned.

1) Collision Checking: To perform a collision check, we must identify trajectories that would place the robot arm with its extruder in collision with either the the partially fabricated object, another of the system's robot arms, or the heated extrusion bed. Collision with previously extruded contours is a 


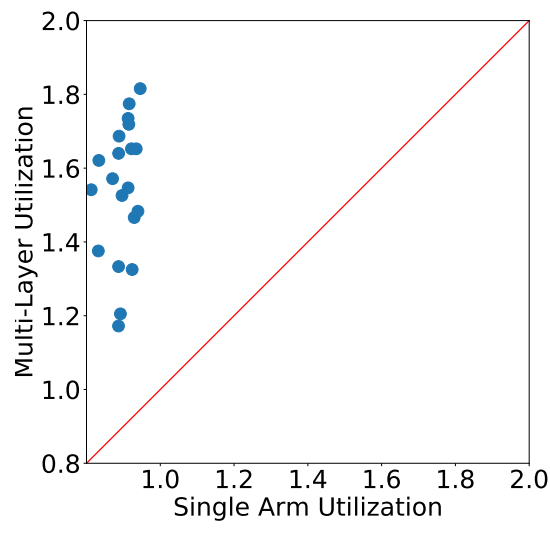

(a) Proposed vs. single arm

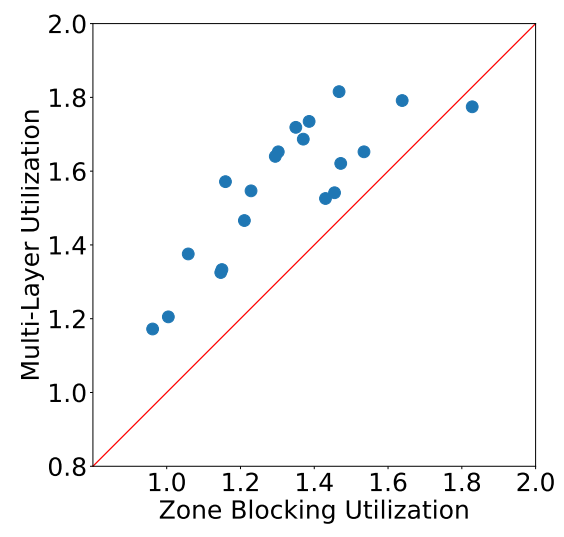

(b) Proposed vs. zone blocking

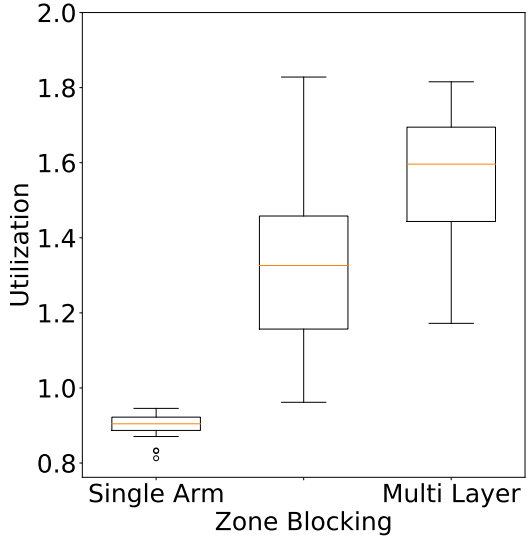

(c) Utilization comparison over 20 benchmark models

Fig. 3. Comparison of utilization per model of our multi-layer approach against the (a) single-arm approach and against (b) zone blocking. Overall we achieve an improvement of $76 \%$ in utilization over a single-arm approach and $20 \%$ over zone blocking.

risk in single-extruder toolpath planning as well, but typically is avoided by adopting the layer-by-layer approach. Any contour in a given layer can be extruded safely if all previously extruded contours lie in the layers below. To avoid collision between an extruder and previously extruded contours, we adopt the approach introduced in our earlier work [7] that uses the dependency graph to identify sets of feasible contours at any intermediate point in the fabrication process. Construction of the dependency graph considers the extruder's geometrical properties (e.g., extruder head size and shape) and thus the feasible contours can be extruded without danger of collision with others.

Checking for collision with other arms is more difficult because it is necessary to consider their possible states at any point in the fabrication process, and these states are not predetermined in our online framework. An exhaustive approach that evaluates all pairs of contours and IK solutions is not computationally feasible since the number of contours is often very large. In general, collision checking is known to be the major computational bottleneck in path planning problems [24]. Thus, any method for planning extruder trajectories must utilize highly efficient collision checking.

Our approach is to utilize the sets of candidate trajectories that are computed during preprocessing. Suppose we have two candidate trajectories $\hat{\mathbf{x}}_{c}^{1}$ and $\hat{\mathbf{x}}_{c^{\prime}}^{2}$ from two robot arms 1 and 2. To determine whether $\hat{\mathbf{x}}_{c}^{1}$ and $\hat{\mathbf{x}}_{c^{\prime}}^{2}$ are in collision, we must consider the 3D geometry of each arm as it follows its trajectory. The IK solutions computed earlier are beneficial here because computing joint positions is then a simple forward kinematics problem. We approximate the shape of individual links using a simple capsule primitive. Likewise, we approximate the print bed using a prism and a the material printed so far using a bounding box in order to consider collisions with those elements. Efficient procedures to check for collisions between these primitive shapes are well known and can be solved in analytical form. Figure 4 demonstrates how collision is checked using forward kinematics (FK) poses: gray cylinders for arm links, green bounding box for extruders,

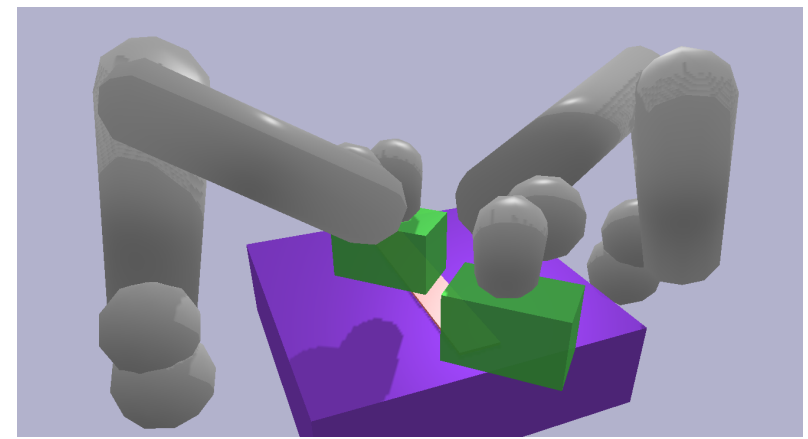

Fig. 4. Visualization of pyBullet [25] 3D collision checking environment showing arms in grey, extrusion end effector in green, print bed in purple and bounding box of completed layers in red.

red bounding box for fabricated contours, and purple box for the print bed.

Collision checking is performed as-needed in the planning component of the framework. Its performance in practice is sufficient to allow the planner to evaluate the possible trajectories when making this choice.

\section{Actuation}

The planner uses the positions of the arms relative to the print bed as calculated by a calibration procedure described in Section VI-B The trajectory for each arm is executed by a controller that tracks its position over time. The joint velocities of each arm are managed such that the trajectories are accurately followed. As this is a closed system with all collision objects being considered by the planner, additional sensors are not required.

\section{Analysis and Discussion}

This algorithmic framework has several properties that promote computational efficiency and utilization. First, our approach eliminates the need to explicitly construct the full (joint) configuration space, which would grow exponentially in the number of arms. In our approach, the computational cost 
of the greedy planner is linear in the number of contours, while the required collision checking is quadratic in the number of extruders. Since the number of extruders is typically small, the overall time required for toolpath construction scales well and is small compared to, for example, actual fabrication time.

Our approach also naturally handles certain delays and disturbances that could arise in practice. For example, it is possible that one extruder moves slightly slower than others. If trajectories are generated offline, this timing mismatch would accumulate over time and possibly lead to unplanned collisions. Our approach is less sensitive to poor time synchronization because collision checking is performed immediately prior to trajectory execution.

Finally, our method favors utilization by considering the maximal set of available contours when making an assignment, instead of restricting this choice to contours within the current layer. Providing more options in this way decreases the likelihood that an extruder is forced to wait for its next assignment. We show in subsequent sections that these benefits lead to notable performance improvements experimentally.

\section{VAlidation With BenchmaRK MOdels IN SIMULATION}

We performed experiments in simulation to evaluate the performance of our framework using a benchmark set of 20 models taken from our prior work [20]. We compare our method to a typical single-extruder approach and to a multiextruder zone-blocking method. The single-extruder approach greedily selects contours by choosing the closest starting point in joint space. Contour choice is limited to the current layer and each layer must be completed before moving to the next. The zone blocking approach divides the workspace equally into two zones (one for each arm) and allocates contours to arms based on its associated zone. Contours that span across both zones are allocated to the starting zone. Contour selection is performed as in the single-extruder approach. Likewise, the zone blocking method must complete a given layer before moving to the next.

Models for the benchmark were chosen manually so as to provide a wide range of potential concurrency. Concurrency potential was estimated by examining contour density within each layer. Models with a predominate pattern of filled regions separated by free space were judged to have a higher potential for concurrency. Models with sufficient separation distance can be viewed as a collection of independent parts in a given layer. All models were centered and scaled to match the physical parameters of our experimental system. We used a single trial of each of the three methods (single arm, zone blocking, multi-layer) to perform toolpath planning for each model. Performance results for each method are shown in Fig. 3 . The single-extruder approach achieved a median utilization of 0.90 , while zone blocking achieved 1.33 and our approach achieved 1.60. Notably, our multi-layer approach outperformed zone blocking in 19 out of the 20 benchmark models with a median increase in utilization of 0.27 (Fig. 3 3 ). This improvement is equivalent to reducing the median print time to $55.6 \%$ of the time taken by the single extruder. In comparison, zone blocking achieves a median print time of $68.1 \%$ of the time taken by a single extruder.

Our approach exhibited two substantial benefits over zone blocking which contribute to its overall performance improvement. Figures 5 (a) and (b) show a representative example of a model in which these two advantages are evident. The first benefit is that our method may choose from among all contours and is not limited to those within its pre-defined zone. For models with an uneven distribution of contours across zones, zone blocking delays the fabrication of contours which, in our method, could be fabricated by another extruder. The model shown in Figs. 5 (a) and (b) illustrates this behavior; the interface between the two zones leads to low utilization because fabrication effectively must alternate between the two arms. More precisely, we found that the performance of zone blocking has a moderate negative correlation $(r=-0.5)$ with the percentage of contours that cut across zones.

The only instance in which zone blocking outperforms our multi-layer approach is a model that contains four completely separate objects, with no contours near the line dividing the two zones. This example is shown in Figs. 5 (c) and (d). This model is a special case where the zone blocking solution amounts to parallel production by two independent singleextruder systems.

Another benefit of our multi-layer approach is that it provides extruders with a larger set of contours to draw from, and thus reduces the need for extruders to go into a waiting state. This benefit is evident in the vertical spines of the model shown in Fig. 5(b). Our approach seemingly switches arbitrarily between spines, but this pattern works to minimize downtime and achieves high utilization (1.82 for multi-layer and 1.47 for zone blocking).

To further examine this effect, we considered the case where our method is limited to single-layer planning for all models in the benchmark. We found that a single-layer planner achieves a lower utilization than multi-layer, reducing median utilization from 1.60 to 1.49 overall.

\section{HARDWARE EXPERIMENTS}

This section presents results from experiments in which we fabricated physical objects. We present the setup of our twoarm system followed by a discussion of fabrication time and quality.

\section{A. Hardware Setup}

The experimental system consists of two 6-DOF UR5e robotic arms [8] placed on opposite ends of an aluminum heated print bed. The UR5e arms have a pose repeatability of $+/-0.03 \mathrm{~mm}$. This level of precision is suitable for use in coarse-resolution FFF systems. The heated bed is constructed from 9 smaller elements arranged in a square grid and linked to a common controller with reference temperature set to $75^{\circ} \mathrm{C}$.

An off-the-shelf extrusion subsystem is mounted as the endeffector of each arm using a custom bracket. Spooled filament is mounted behind each arm. The extrusion system uses a $1.2 \mathrm{~mm}$ diameter heated nozzle extruding PLA (Polylactic Acid) at 200C. Filament is pushed through the heated nozzle 


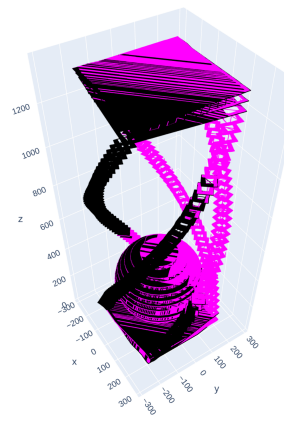

(a) Zone blocking, $\sigma=1.47$

(b) Multi-layer, $\sigma=1.82$

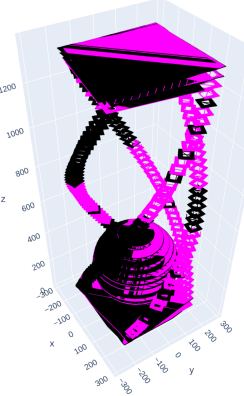

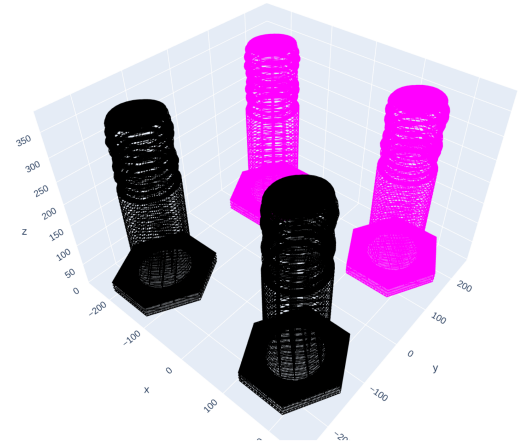

(c) Zone blocking, $\sigma=1.83$

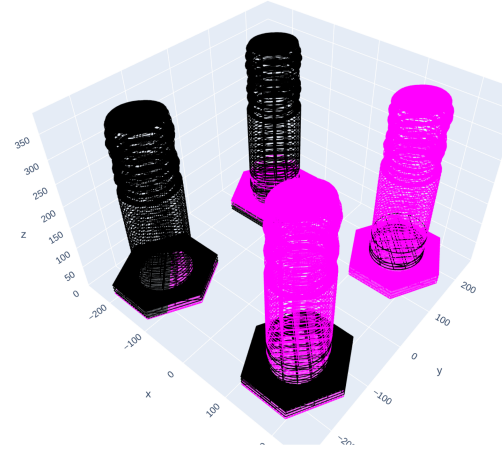

(d) Multi-layer, $\sigma=1.77$

列 of 1.47, (b) proposed multi-layer approach with utilization of 1.82. The special case where zone blocking marginally outperforms our method, achieving a utilization of 1.83 (c), while our approach (d) achieves a utilization of 1.77.
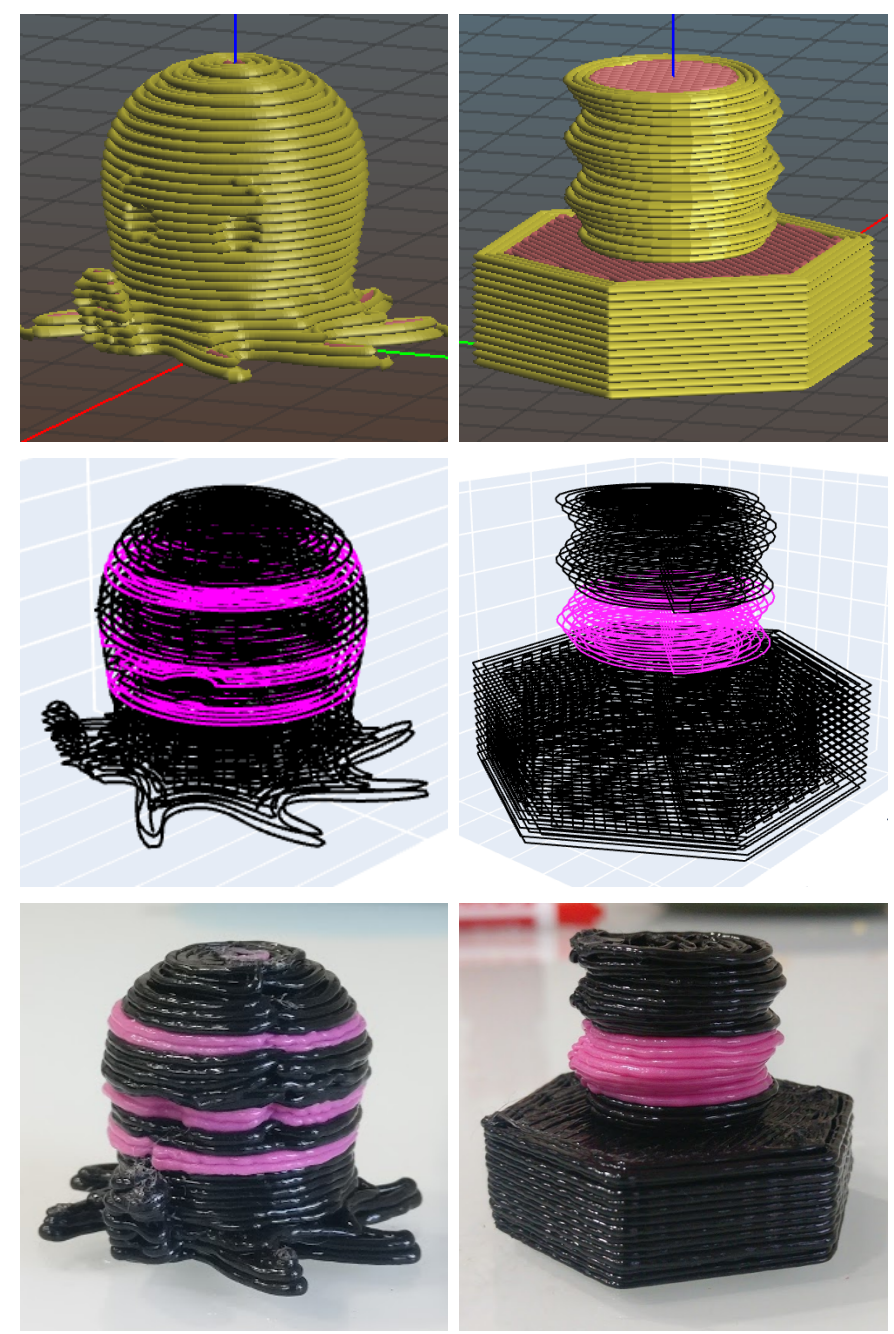

Fig. 6. Example parts showing sliced models (top row), scheduled models (middle row), fabricated models (bottom row). Three copies of each model were printed simultaneously, forcing arms to work on the same model. Deposition by arm 1 in black, and by arm 2 in pink.

by an embedded gear in the extruder. This $1.2 \mathrm{~mm}$ nozzle extrudes material in thicker tracks than most desktop 3D printers, but is a common size used in industrial-scale FFF systems. This size of extrusion width allows for minor arm vibration without significant distortion to the shape of the extruded track and is consistent with other systems with large workspaces that prioritize deposition speed over fine detail.

\section{B. Calibration Procedure}

The position and orientation of the arms relative to the print bed $P_{B A}$ and the position and orientation of the extruder tip relative to the end of the arm $P_{A E}$ are initially unknown. To compute both of these transforms simultaneously, the arms are moved to 11 marked positions on the print bed with known coordinates in the print bed frame, and joint angles are recorded for each marked position. Given the joint angles $j$ of the arms, $P_{B A}$ and $P_{A E}$, the position of the the extruder tip can be calculated using forward kinematics $f k\left(j, P_{B A}, P_{A E}\right)$. A least-squares solver is used to minimize the error between the print bed coordinates and the computed extruder tip position by changing $P_{B A}$ and $P_{A E}$. To correctly attribute transformation differences between $P_{B A}$ and $P_{A E}$, the extruder tip must reach two of the marked positions with different orientations.

\section{Results}

We fabricated several models chosen from the benchmark to demonstrate accurate calibration and collision avoidance, and also realize the speed improvements achieved by our multilayer planner. A large object spanning the print bed is shown in Fig. 1. The arms did not collide, and their relative positions matched the simulated trajectory. The fabricated models show that the calibration and execution were sufficiently accurate to produce objects of good quality. Models fabricated in this experiment have a layer height of $1 \mathrm{~mm}$ and a measured height of $.99 \pm 0.05 \mathrm{~mm}$, as expected. Contour extrusion width is also an important control parameter; inspected layers have an expected contour width of $1.35 \mathrm{~mm}$ and a measured width of $1.33 \pm 0.09 \mathrm{~mm}$.

Execution time closely matched the simulation time. Minor delays caused by variations in communication latency were successfully handled such that the maximum synchronization error between arms was $0.1 \mathrm{~s}$.

Extrusion of thermoplastics for FFF requires balancing the deposition rate and temperature to ensure solidification and to 
reduce common fabrication failures categorized in [26]. Our system quality and accuracy is susceptible to these same potential failure modes. Specifically, we observed stringing, overextrusion and warping. Stringing was addressed by increasing the retraction distance while traveling between contours. Both warping and over-extrusion were addressed with solutions widely used in desktop 3D printers in thermal and extrusion parameter tuning. These issues are not related to the two arm coordination nature of the system.

Two examples of sliced, scheduled, and fabricated components are provided in Fig 6 , where the two colors indicate which arm deposited the contours (black for arm 1 and pink for arm 2). The fabricated components show excellent agreement with the deposition plan and comparable quality to commercial FFF systems.

\section{CONCLUSION}

In this paper we have presented and implemented a system for collaborative multi-extruder fused filament fabrication. The approach presented in this paper significantly improves upon existing methods in terms of utilization (and thus fabrication speed). Moreover, due to our novel online approach to collision checking our approach does not require any a priori segmentation of the model or specification of workspaces. Existing methods relying on segmentation of blocking essentially split the model physically, deviating from the original design and potentially creating part quality issues.

We believe that our approach provides a starting point for several interesting new lines of inquiry. A natural extension the approach presented here is to incorporate more than two extruders. Our algorithm extends to the case of an arbitrary number of extruders; we plan to conduct comparative studies that characterize the natural trade-off between collisions and utilization in a fixed size build area. In small models, an additional extruder does not provide much benefit as there is little concurrent fabrication possible. Investigation into this trade-off may yield an optimum system design based on part size and/or geometry.

Our approach is based on a set of input contours generated from existing two-dimensional slicing methods, such as Sli3r [23]. Intuitively, our multi-layer approach to toolpath construction succeeds because we are able to minimize wasted motion by working in all three dimensions. This raises the question of whether two-dimensional slicing hinders toolpath quality. In ongoing work, we are exploring a new approach to slicing and contour generation in three dimensions as a way to address this question.

Finally we are also exploring a more powerful algorithmic framework for multi-extruder toolpath planning. We recently applied a Monte Carlo tree search (MCTS) framework to toolpath planning [21] that makes use of the decentralized Monte Carlo Tree Search (Dec-MCTS) [27] algorithm to perform decentralized coordinated planning of each arm/extruder. Dec-MCTS is designed to find globally optimal solutions and has been shown to be far more computationally efficient than an equivalent centralized approach for coordinated planning problems. We are currently exploring how Dec-MCTS can be applied to coordinated toolpath planning with multiple extruders in order to further increase utilization.

\section{REFERENCES}

[1] ISO/TC 261 Additive manufacturing, ISO/ASTM 52900:2015 Additive Manufacturing - General Principles - Terminology. ISO, 2015.

[2] F. Wang, S. Fathizadan, F. Ju, K. Rowe, and N. Hofmann, "Print surface thermal modeling and layer time control for large-scale additive manufacturing," IEEE Trans. Autom. Sci. Eng., vol. 18, no. 1, pp. 244-254, 2021.

[3] Y. Wang, Z. Gu, L. Song, T. Li, H. Cui, and F. C. M. Lau, "Speeding up 3D printing using multi-head slicing algorithms," in Proc. of IEEE ES, Sep. 2017, pp. 99-106.

[4] Z. Jiang, S. Psulkowski, A. Nwodu, H. Wang, and T. Dickens, "Improved co-scheduling of printing path scanning for collaborative additive manufacturing," in Proc. of ASME MSEC, 2019.

[5] H. Bui, H. A. Pierson, S. G. Nurre, and K. M. Sullivan, "Tool path planning optimization for multi-tool additive manufacturing," in Proc. of ICPR, 2019.

[6] T. Yao, K. Zhang, Z. Deng, and J. Ye, "A novel generalized stress invariant-based strength model for inter-layer failure of FFF 3D printing PLA material," Mater. Des., vol. 193, Aug. 2020.

[7] S. Lensgraf and R. R. Mettu, "Beyond layers: A 3Daware toolpath algorithm for fused filament fabrication," in Proc. of IEEE ICRA, 2016, pp. 3625-3631.

[8] "UR5 collaborative robot arm," https://www. universal-robots.com/products/ur5-robot, accessed: 2021-03-01.

[9] P. Nicholas, G. Rossi, E. Williams, M. Bennett, and T. Schork, "Integrating real-time multi-resolution scanning and machine learning for conformal robotic $3 \mathrm{~d}$ printing in architecture," International Journal of Architectural Computing, 2020.

[10] C. Gosselin, R. Duballet, P. Roux, N. Gaudillière, J. Dirrenberger, and P. Morel, "Large-scale 3d printing of ultra-high performance concrete-a new processing route for architects and builders," Materials \& Design, vol. 100, pp. 102-109, 2016.

[11] H. Shen, L. Pan, and J. Qian, "Research on large-scale additive manufacturing based on multi-robot collaboration technology," Addit. Manuf., vol. 30, 2019.

[12] P. M. Bhatt, R. K. Malhan, A. V. Shembekar, Y. J. Yoon, and S. K. Gupta, "Expanding capabilities of additive manufacturing through use of robotics technologies: A survey," Addit. Manuf., vol. 31, Jan. 2020.

[13] K. McQueen, S. Darensbourg, C. Moore, T. Dickens, and C. Allen, "Efficient path planning of secondary additive manufacturing operations," MATEC Web Conf., vol. 249, p. 03011, 2018.

[14] Y. Cai and S. Choi, "Deposition group-based toolpath planning for additive manufacturing with multiple robotic actuators," Procedia Manuf., vol. 34, pp. 584-593, 2019.

[15] C. Chang, M. J. Chung, and B. H. Lee, "Collision avoidance of two general robot manipulators by minimum 
delay time," IEEE Trans. Syst. Man Cybern., vol. 24, no. 3, pp. 517-522, 1994.

[16] P. A. O’Donnell and T. Lozano-Pérez, "Deadlock-free and collision-free coordination of two robot manipulators," in Proc. of IEEE ICRA, 1989, pp. 484-489.

[17] E. K. Xidias, P. T. Zacharia, and N. A. Aspragathos, "Time-optimal task scheduling for two robotic manipulators operating in a three-dimensional environment," $J$. Syst. Contr. Eng., vol. 224, no. 7, pp. 845-855, 2010.

[18] P. Bosscher and D. Hedman, "Real-time collision avoidance algorithm for robotic manipulators," Ind. Robot., vol. 38, no. 2, pp. 186-197, 2011.

[19] S. Lensgraf and R. R. Mettu, "An improved toolpath generation algorithm for fused filament fabrication," in Proc. of IEEE ICRA, 2017, pp. 1181-1187.

[20] — , "Incorporating kinematic properties into fused deposition toolpath optimization," in Proc. of IEEE/RSJ IROS, 2018, pp. 8622-8627.

[21] C. Yoo, S. Lensgraf, L. Clemon, R. Fitch, and R. R. Mettu, "Toward optimal FDM toolpath planning with Monte Carlo tree search," in Proc. of IEEE ICRA, 2020.

[22] Y. Jin, H. A. Pierson, and H. Liao, "Toolpath allocation and scheduling for concurrent fused filament fabrication with multiple extruders," IISE Trans., vol. 51, no. 2, pp. 192-208, 2019.

[23] "Slic3r gcode generator," http://www.slic3r.org, accessed: 2021-03-01.

[24] L. Janson, E. Schmerling, A. Clark, and M. Pavone, "Fast Marching Tree: A fast marching sampling-based method for optimal motion planning in many dimensions," Int. J. Rob. Res., vol. 34, no. 7, pp. 883-921, 2015.

[25] E. Coumans and Y. Bai, "PyBullet, a python module for physics simulation for games, robotics and machine learning," http://pybullet.org, 2021, accessed: 2021-0301 .

[26] R. Song, L. Clemon, and C. Telenko, "Uncertainty and variability of energy and material use by fused deposition modeling printers in makerspaces," J. Ind. Ecol., vol. 23, no. 3, pp. 699-708, 2019.

[27] G. Best, O. Cliff, T. Patten, R. R. Mettu, and R. Fitch, "Dec-MCTS: Decentralized planning for multi-robot active perception," Int. J. Robot. Res., vol. 38, no. 2-3, pp. 316-337, 2019.

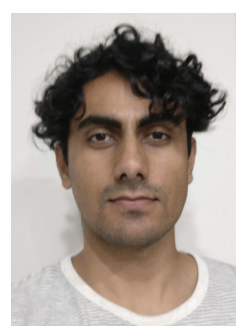

Jayant Khatkar received a B.E. from the University of Sydney. He worked as a data scientist for 1.5 years and is currently pursuing a Ph.D. at the University of Technology Sydney.

His research interests include systems engineering, planning and additive manufacturing.

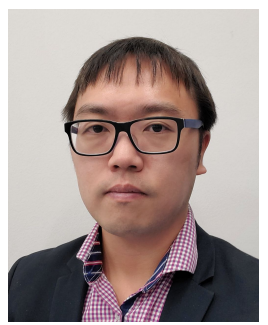

Chanyeol Yoo is a postdoctoral research fellow at UTS with expertise in path planning and formal methods applied to outdoor robotics. Dr. Yoo received his B.Eng (first class honour) in computer systems engineering from the University of Auckland (New Zealand). He completed his Ph.D. at the Australian Centre for Field Robotics at the University of Sydney, and a postdoc with at Boston University (USA) before joining UTS in 2016.

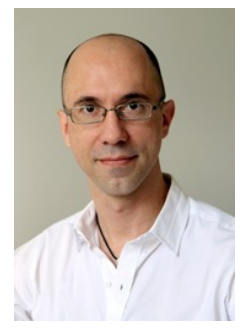

Robert Fitch is Professor and Head of School of Mechanical and Mechatronic Engineering at the University of Technology Sydney. He received his Ph.D. in Computer Science (Robotics) from Dartmouth College in 2005. He was with the Australian Centre for Field Robotics (ACFR) at The University of Sydney before joining UTS in 2016. Prof. Fitch is co-chair of the IEEE RAS Technical Committee on Multi-Robot Systems and is co-founder of the IEEE International Symposium on Multi-Robot and Multi-Agent Systems. His research interests include active perception, information-based motion planning, communication-aware algorithms, and coordinated decision making for robotic systems.

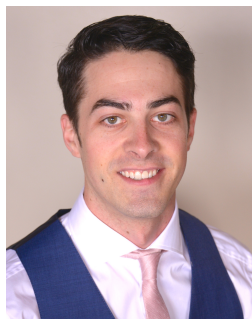

Lee Clemon is a research scientist in advanced manufacturing and high consequence design and licensed professional engineer. Dr. Clemon received his B.S. in Mechanical engineering from the University of Kansas and his Ph.D. from the University of California, Berkeley. His current research interests are in process improvement and material property manipulation in advanced manufacturing processes, with an emphasis on additive and hybrid additivesubtractive manufacturing through particulate, wire, layer, and ensemble fabrication methods. Dr. Clemon is a Lecturer at the University of Technology Sydney, in the School of Mechanical and Mechatronic Engineering, a management member of the Centre for Advanced Manufacturing, and member of the RF and Communication Technologies Lab. 


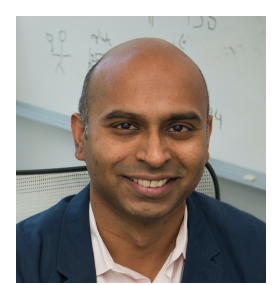

Ramgopal Mettu is an Associate Professor in the Department of Computer Science at Tulane University. He received his B.S. degree in Computer Science from the University of Texas at Austin in 1997, and his Ph.D. in Computer Science in 2002. He held a postdoctoral research associate position at Dartmouth College from 2002 to 2005 and was subsequently a faculty member in the ECE Department at UMass Amherst until 2011. Dr. Mettu's research focus is the design and analysis of algorithms for a variety of application areas. In the areas of robotics and additive manufacturing, his primary interest in developing rigorous algorithmic approaches for multi-robot systems and optimizing the speed and strength of 3D printing. 